\title{
Passivation of Electron Trap States in InP Quantum Dots with Benzoic Acid Ligands
}

\author{
Xingao Zhang, Margaret H. Hudson and Felix N. Castellano* \\ Department of Chemistry, North Carolina State University, Raleigh, North Carolina 27695-8204, \\ United States \\ e-mail : fncastel@ncsu.edu
}
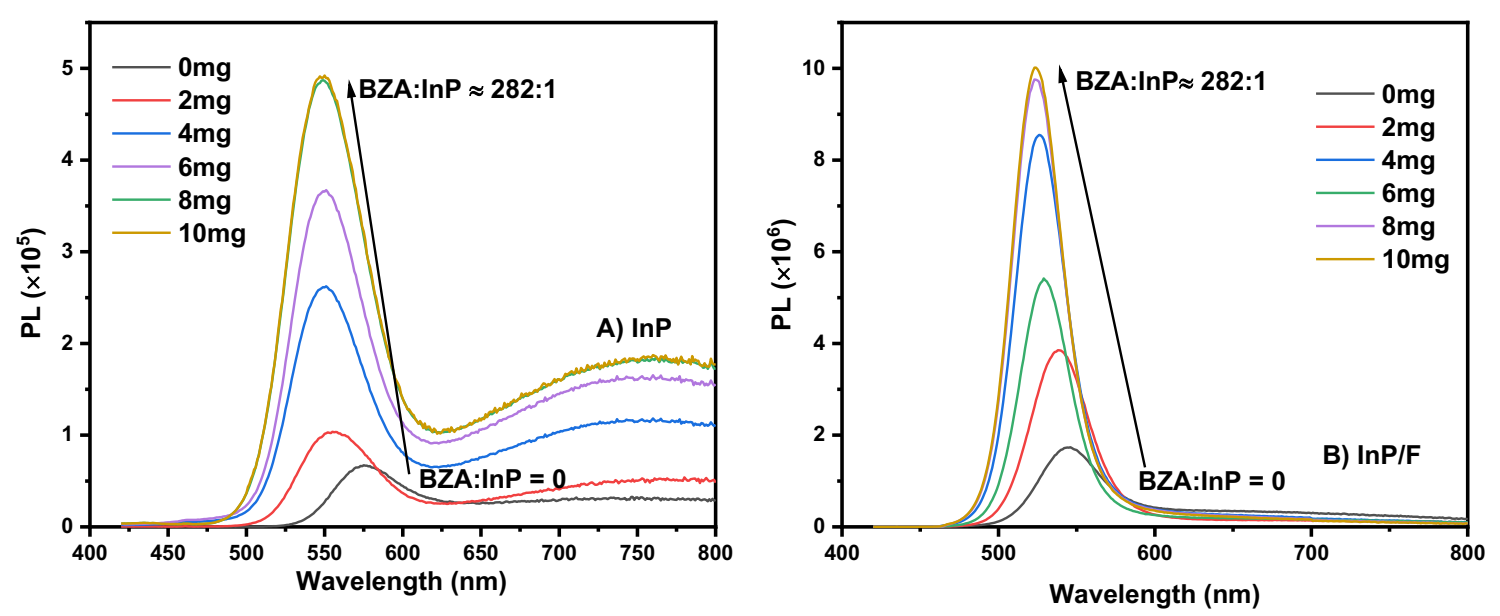

Figure S1. PL spectra of BZA-treated InP QDs A) InP QDs, B) InP/F QDs in toluene solvent at increasing the ratio of BZA to InP QDs from 0 to 282. The PL spectra demonstrate that $8 \mathrm{mg}$ BZA (BZA: InP QDs $\approx 225: 1$ ) is able to maximize the band-edge PL of in both InP QDs and InP/F QDs. 

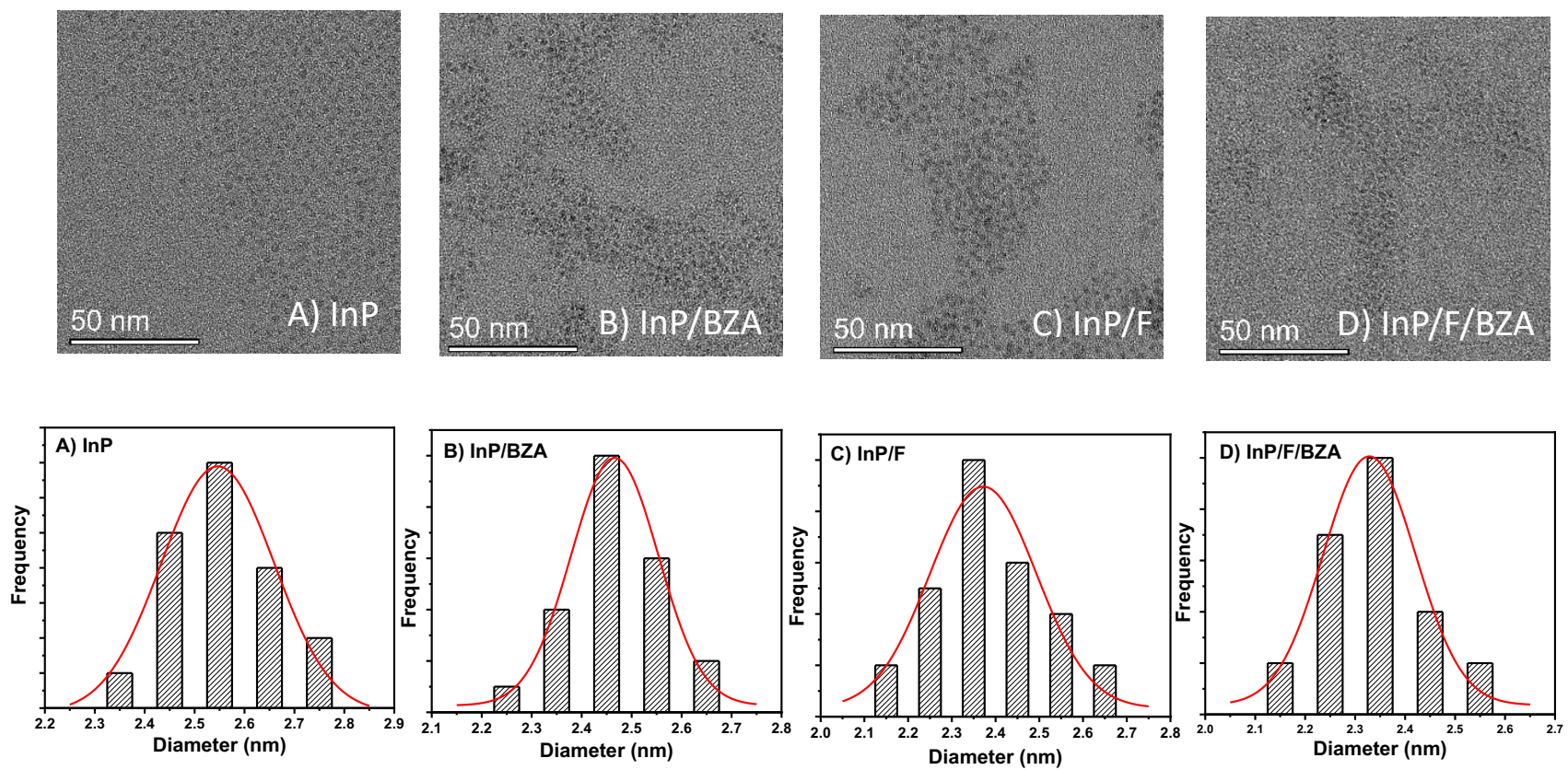

Figure S2. TEM images and size distribution histogram for TEM images of A) InP QDs, $\bar{d}=$ $2.54 \pm 0.20 \mathrm{~nm}, \mathrm{~B}) \mathrm{InP} / \mathrm{BZA}$ QDs, $\bar{d}=2.47 \pm 0.20 \mathrm{~nm}, \mathrm{C}) \mathrm{InP} / \mathrm{F}$ QDs, $\bar{d}=2.37 \pm 0.20 \mathrm{~nm}$ and D) InP/F/BZA QDs, $\bar{d}=2.33 \pm 0.20 \mathrm{~nm}$. For each image, 50 particles were measured for the histogram. Each Size distribution histogram is fitted by Gaussian function shown as red line in each graph.

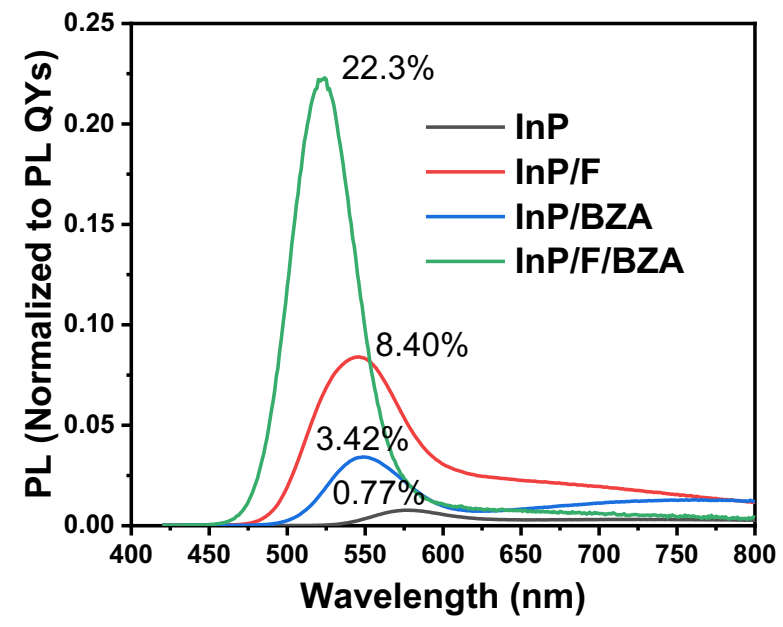

Figure S3. PL spectrum of InP QDs with different surface conditions normalized to each PLQY. The value represents PLQY of each sample. All data were collected in chloroform solvent. (Excitation wavelength $=415 \mathrm{~nm}$ ) 

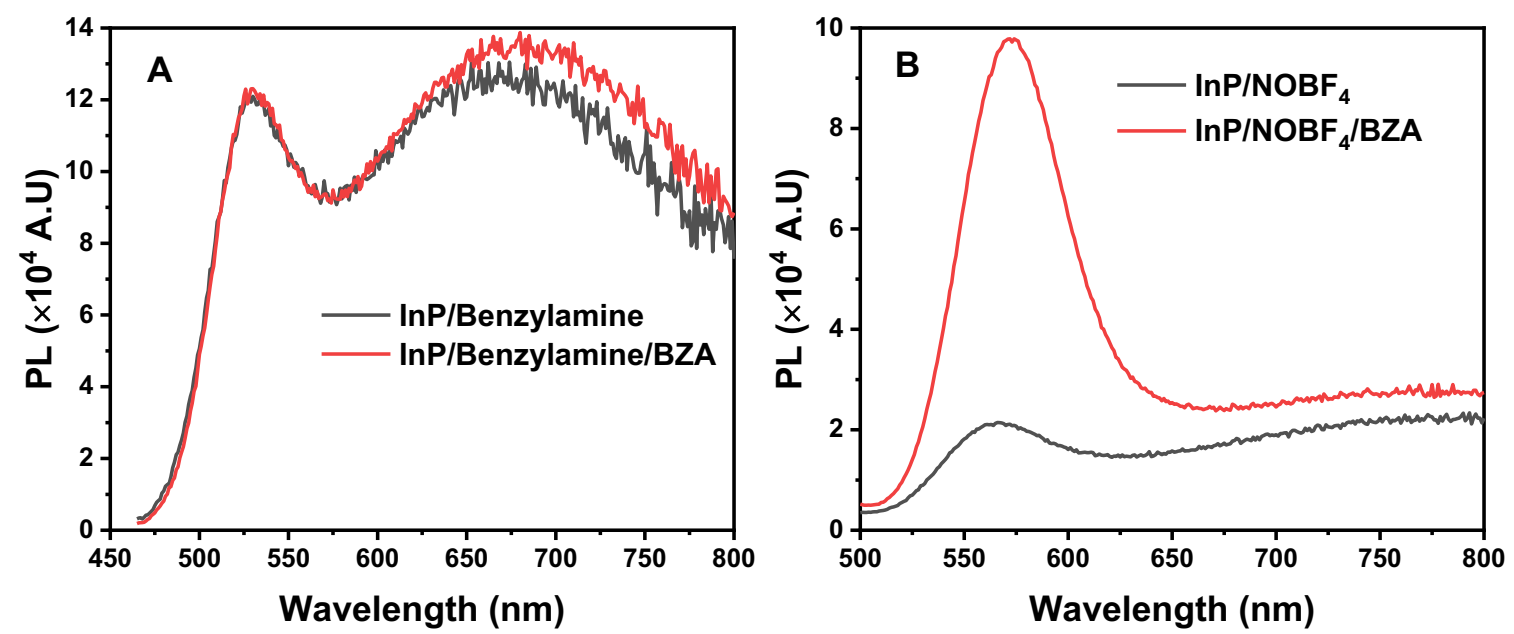

Figure S4. PL spectra (Excitation wavelength $=415 \mathrm{~nm}$ ) of $(\mathrm{A}) \mathrm{InP} /$ Benzylamine and $\mathrm{InP} /$ Benzylamine with $\mathrm{BZA}$ and $(\mathrm{B}) \mathrm{InP} / \mathrm{NOBF}_{4}$ and $\mathrm{InP} / \mathrm{NOBF}_{4}$ with BZA. All data were collected in chloroform solvent.

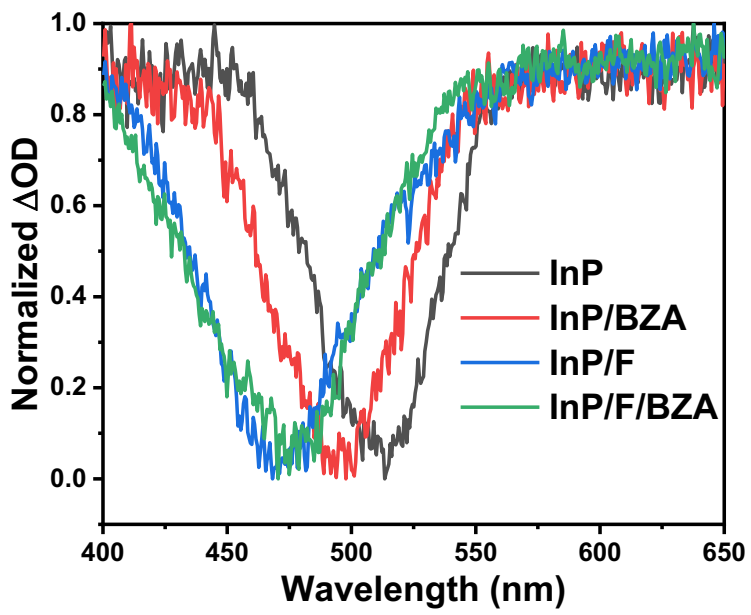

Figure S5. Normalized TA spectra of InP QDs, InP/BZA QDs, InP/F QDs, and InP/F/BZA QDs in chloroform measured at 0ns. (Excitation wavelength, $415 \mathrm{~nm} ; 1 \mathrm{~mJ}$ ) 

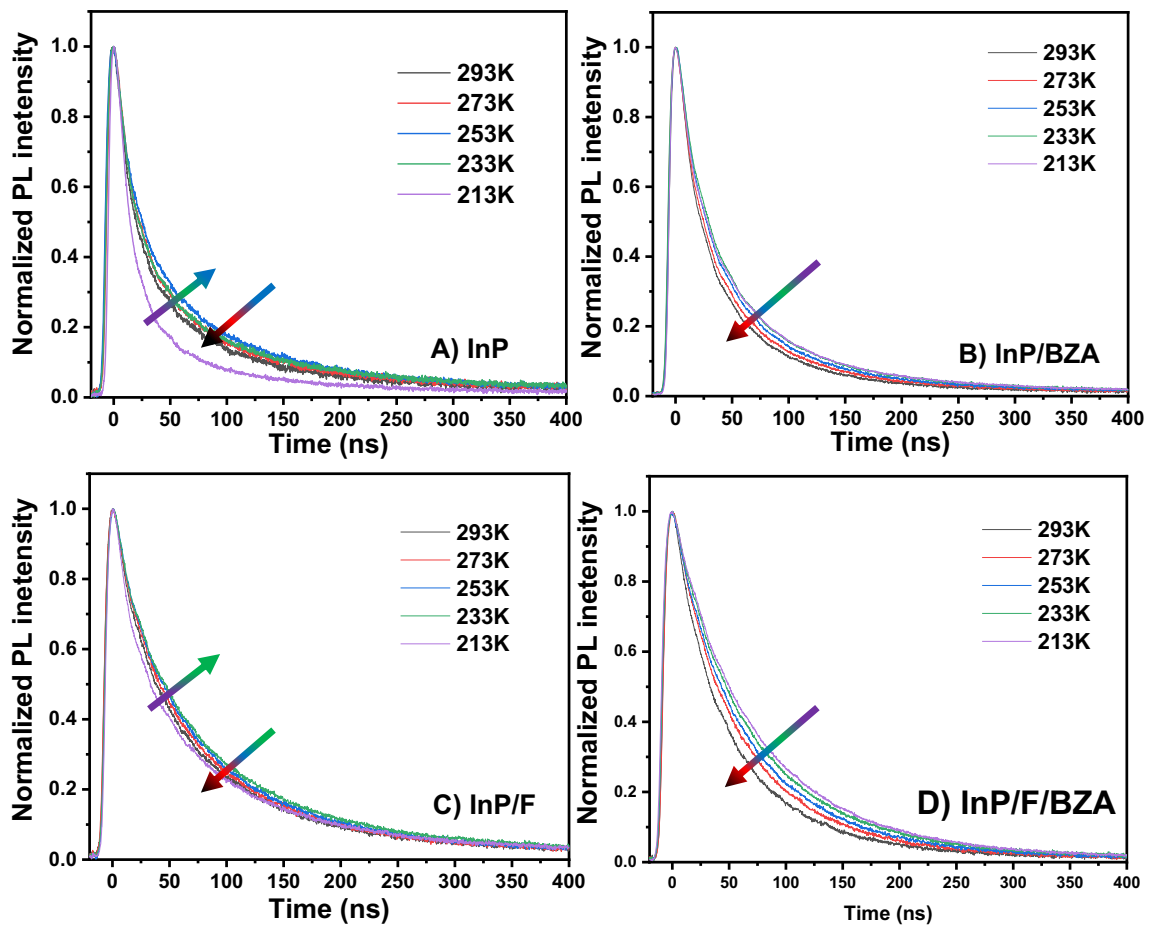

Figure S6. TDPL kinetics of (A) InP QDs, (B) InP/BZA QDs, (C) InP/F QDs and (D) InP/F/BZA QDs. All data were collected from $213 \mathrm{~K}$ to $293 \mathrm{~K}$ in chloroform solvent. (Excitation wavelength $=415 \mathrm{~nm}$ ) Arrows and arrow colors in spectra shows the trends of band-edge PL kinetics change along with temperature change. 


\section{Calculation of InP QDs concentration}

The concentration of the InP QD solution was calculated from the first excitonic absorption peak intensity and position and using the empirical function ${ }^{1}$ and Beer-Lambert law. The diameter was calculated from TEM images. Assuming each QD is a perfect sphere (see TEM images in Figure S2).

$$
\begin{gathered}
\varepsilon=3046.1 \times D^{3}-76532 \times D^{2}+(5.5137 \times 105) \times D-8.9839 \times 10^{5} \\
A b s=\varepsilon \times l \times c \quad \text { S2 }
\end{gathered}
$$

where $\varepsilon$ is the correlation molar extinction coefficient of InP QDs, Abs is absorbance, D is the diameter of InP QDs, 1 is the optical path length in $\mathrm{cm}$ and $\mathrm{c}$ is the concentration of the InP QD solution.

\section{GSB-recovery, PL and trap PL kinetics data fitting}

GSB-recovery and PL kinetics of InP QDs, InP/BZA QDs, InP/F QDs, and InP/F/BZA were fitted by biexponential eq S3.

$$
O D=A_{1} * e^{\left(-\frac{t}{\tau_{1}}\right)}+A_{2} * e^{\left(-\frac{t}{\tau_{2}}\right)}
$$

Weighted lifetimes were calculated by S4.

$$
\bar{\tau}=\frac{\sum_{i} A_{i} \tau_{i}}{\sum_{i} A_{i}}
$$

The $\bar{\tau}$ is the weighted lifetime, $\mathrm{A}_{1}$ and $\mathrm{A}_{2}$ are amplitudes for both lifetimes, $\tau_{1}$ and $\tau_{2}$ are lifetimes.

All unsaturated surface atoms can generate trap states. Thus, the decay rates may be various. Trap PL kinetics were fitted by stretched exponential function eq S5. The weighted lifetimes were calculated by $\mathrm{S} 6$.

$$
O D=A * e^{-\left(\frac{t}{\tau}\right)^{\beta}}
$$

The average weighted lifetime $\bar{\tau}$, was calculated from the following equation,

$$
\bar{\tau}=\frac{\tau}{\beta} \times \Gamma\left(\frac{1}{\beta}\right)
$$

Where $\mathrm{A}$ and $\tau$ are the amplitude and the stretched lifetime, respectively, and $\beta$ is the stretching exponent. 
Table S1. Fitting parameters for GSB-recovery, band-edge PL and trap PL kinetics of representative samples.

\begin{tabular}{|c|c|c|c|c|c|c|}
\hline $\begin{array}{c}\text { GSB-recovery } \\
\text { Kinetics }\end{array}$ & $\tau_{1}(\mathrm{~ns})$ & $\mathrm{A}_{1}$ & $\tau_{2}(\mathrm{~ns})$ & $\mathrm{A}_{2}$ & $\bar{\tau}(\mathrm{ns})$ & R-square \\
\hline $\mathrm{InP}$ & 16.8 & 0.55 & 114.2 & 0.43 & 60 & 0.998 \\
\hline $\mathrm{InP} / \mathrm{BZA}$ & 17.3 & 0.47 & 109.6 & 0.53 & 66 & 0.998 \\
\hline $\mathrm{InP} / \mathrm{F}$ & 22.6 & 0.47 & 127.1 & 0.52 & 77 & 0.998 \\
\hline $\mathrm{InP} / \mathrm{F} / \mathrm{BZA}$ & 28.2 & 0.67 & 125.1 & 0.33 & 60 & 0.979 \\
\hline
\end{tabular}

\begin{tabular}{|c|c|c|c|c|c|c|}
\hline Band-edge PL Kinetics & $\tau_{1}(\mathrm{~ns})$ & $\mathrm{A}_{1}$ & $\tau_{2}(\mathrm{~ns})$ & $\mathrm{A}_{2}$ & $\bar{\tau}(\mathrm{ns})$ & R-square \\
\hline $\mathrm{InP}$ & 17.0 & 0.66 & 94.7 & 0.35 & 44 & 0.999 \\
\hline $\mathrm{InP} / \mathrm{BZA}$ & 18.1 & 0.6 & 66.5 & 0.44 & 39 & 0.999 \\
\hline $\mathrm{InP} / \mathrm{F}$ & 25.5 & 0.43 & 99.5 & 0.57 & 68 & 0.999 \\
\hline $\mathrm{InP} / \mathrm{F} / \mathrm{BZA}$ & 33.6 & 0.53 & 81.6 & 0.49 & 57 & 0.999 \\
\hline
\end{tabular}

\begin{tabular}{|c|c|c|c|c|}
\hline Trap PL Kinetics & $\tau(\mathrm{ns})$ & $\beta$ & $\bar{\tau}(\mathrm{ns})$ & R-square \\
\hline InP & 86.9 & 0.65 & 119 & 0.992 \\
\hline InP/BZA & 74.5 & 0.62 & 108 & 0.993 \\
\hline $\mathrm{InP} / \mathrm{F}$ & 70.6 & 0.55 & 120 & 0.999 \\
\hline $\mathrm{InP} / \mathrm{F} / \mathrm{BZA}$ & 71.6 & 0.59 & 110 & 0.999 \\
\hline
\end{tabular}




\section{PL kinetics of InP QDs data fitting}

Decay kinetics of InP QDs, InP/BZA QDs, InP/F QDs, and InP/F/BZA are fitted by biexponential eq S3. Weighted lifetimes were calculated by S4.

Table S2. Fitting parameters for TDPL kinetics.

\begin{tabular}{|c|c|c|c|c|c|c|c|}
\hline Sample & $\begin{array}{c}\text { Temperature } \\
(\mathrm{K})\end{array}$ & $\mathrm{A}_{1}$ & $\tau_{1}(\mathrm{~ns})$ & $\mathrm{A}_{2}$ & $\tau_{2}(\mathrm{~ns})$ & $\bar{\tau}(\mathrm{ns})$ & $\mathrm{R}$-square \\
\hline \multirow{5}{*}{ InP } & 213 & 0.88 & 15.2 & 0.19 & 97.0 & 30 & 0.999 \\
\hline & 233 & 0.67 & 18.7 & 0.35 & 112.0 & 51 & 0.999 \\
\hline & 253 & 0.60 & 19.7 & 0.40 & 109.0 & 55 & 0.999 \\
\hline & 273 & 0.66 & 18.9 & 0.36 & 102.1 & 48 & 0.999 \\
\hline & 293 & 0.66 & 17.0 & 0.35 & 94.7 & 44 & 0.999 \\
\hline \multirow{5}{*}{ InP/BZA } & 213 & 0.46 & 18.4 & 0.54 & 74.0 & 48 & 0.999 \\
\hline & 233 & 0.53 & 22.5 & 0.49 & 76.3 & 48 & 0.999 \\
\hline & 253 & 0.55 & 21.2 & 0.48 & 72.8 & 45 & 0.999 \\
\hline & 273 & 0.56 & 19.3 & 0.46 & 69.9 & 42 & 0.999 \\
\hline & 293 & 0.60 & 18.2 & 0.44 & 66.5 & 39 & 0.999 \\
\hline \multirow{5}{*}{$\mathrm{InP} / \mathrm{F}$} & 213 & 0.48 & 20.7 & 0.53 & 106.6 & 66 & 0.999 \\
\hline & 233 & 0.41 & 29.7 & 0.58 & 111.6 & 78 & 0.999 \\
\hline & 253 & 0.43 & 30.3 & 0.57 & 107.8 & 74 & 0.999 \\
\hline & 273 & 0.43 & 28.5 & 0.57 & 103.3 & 71 & 0.999 \\
\hline & 293 & 0.43 & 25.5 & 0.57 & 99.5 & 68 & 0.999 \\
\hline \multirow{5}{*}{$\mathrm{InP} / \mathrm{F} / \mathrm{BZA}$} & 213 & 0.41 & 45.5 & 0.59 & 97.9 & 76 & 0.999 \\
\hline & 233 & 0.44 & 43.2 & 0.56 & 94.6 & 72 & 0.999 \\
\hline & 253 & 0.44 & 38.7 & 0.56 & 89.0 & 67 & 0.999 \\
\hline & 273 & 0.46 & 34.6 & 0.56 & 89.2 & 65 & 0.999 \\
\hline & 293 & 0.53 & 33.6 & 0.49 & 81.6 & 57 & 0.999 \\
\hline
\end{tabular}

(1) Talapin, D. V. Experimental and Theoretical Studies on the Formation of Highly Luminescent II-VI, III-V and Core-Shell Semiconductor Nanocrystals. Ph.D. Dissertation, University of Hamburg, Hamburg, Germany, 2002. 